\title{
A Face of a Projective Triangulation Removed for Its Geometric Realizability
}

\author{
Atsuhiro Nakamoto • Shoichi Tsuchiya
}

Received: 6 September 2010 / Revised: 18 July 2011 / Accepted: 21 July 2011 /

Published online: 18 August 2011

(C) Springer Science+Business Media, LLC 2011

\begin{abstract}
Let $M$ be a map on a surface $F^{2}$. A geometric realization of $M$ is an embedding of $F^{2}$ into a Euclidian 3-space $\mathbb{R}^{3}$ with no self-intersection such that each face of $M$ is a flat polygon. In Bonnington and Nakamoto (Discrete Comput. Geom. 40:141-157, 2008), it has been proved that every triangulation $G$ on the projective plane has a face $f$ such that the triangulation $G-f$ on the Möbius band obtained from $G$ by removing the interior of $f$ has a geometric realization. In this paper, we shall characterize such a face $f$ of $G$.
\end{abstract}

Keywords Geometric realization · Triangulation · Projective plane · Möbius band

\section{Introduction}

A map $M$ means a fixed embedding of a simple graph on a surface $F^{2}$. We only deal with 2-cell embedding, that is, one all of whose faces are homeomorphic to 2-cells. A triangulation on a surface $F^{2}$ is a map on $F^{2}$ such that each face is bounded by a 3-cycle, where a $k$-cycle means a cycle of length $k$. For a map $M$ on a surface, a cycle $C$ is said to be essential if $C$ does not bound a 2-cell on the surface, and it is trivial otherwise. (Similarly, we define essential and trivial closed curves on the surface.) For a face or a 2-cell region $R$ of a map $M$, let $\partial R$ denote the boundary

\footnotetext{
A. Nakamoto

Department of Mathematics, Faculty of Education and Human Sciences, Yokohama National University, 79-2 Tokiwadai, Hodogaya-ku, Yokohama 240-8501, Japan

e-mail: nakamoto@ynu.ac.jp

S. Tsuchiya $(\bowtie)$

Department of Information Media and Environment Sciences, Graduate School of Environment and Information Sciences, Yokohama National University, 79-7 Tokiwadai, Hodogaya-ku, Yokohama 240-8501, Japan

e-mail: s-s-t-b@mail.goo.ne.jp
} 
Fig. 1 A Möbius triangulation with no geometric realization constructed by Brehm

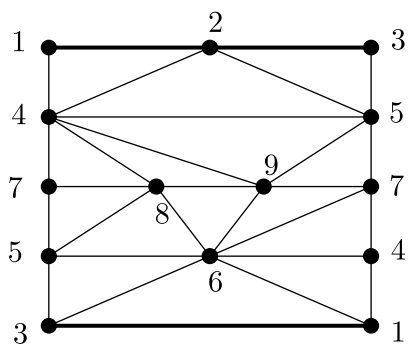

closed walk of $R$. For a cycle $C$ of $M$ bounding a 2-cell, let Int $C$ denote the subgraph of $G$ consisting of all vertices and edges in the interior and the boundary of $C$. On the other hand, let int $C=\operatorname{Int} C-V(C)$. We say that a vertex and an edge of int $C$ are inner. Let $C$ and $D$ be trivial cycles of $M$. We say that $C$ surrounds $D$ if $\operatorname{Int} C$ contains $D$. In particular, if int $C$ contains $D$, then $C$ is a nesting cycle of $D$, which is an important definition in our paper. We often denote vertices and edges by symbols with subscripts, in which we take suitable modulus.

Let $M$ be a map on a surface $F^{2}$. A geometric realization of $M$ is an embedding of $F^{2}$ into a Euclidian 3-space $\mathbb{R}^{3}$ with no self-intersection such that each face of $M$ is a flat polygon. Steinitz [11] has proved that a spherical map has a geometric realization as a convex polyhedron if and only if its graph is 3-connected. (The 3connectedness is necessary to make a geometric realization as a convex polygon. However, our definition of a geometric realization allows two adjacent faces to lie on the same plane in $\mathbb{R}^{3}$.) Moreover, Archdeacon, Bonnington, and Ellis-Monaghan [1] have proved that every toroidal triangulation has a geometric realization. In general, Grünbaum has conjectured that every triangulation on any orientable closed surface has a geometric realization [6]. However, Bokowski and Oliveira [3] have shown that there exists a triangulation on the orientable closed surface of genus six which has no geometric realization. Moreover, Schewe [10] has shown that there exists a triangulation on the orientable closed surface of genus five which has no geometric realization. Hence Grünbaum's conjecture is no longer true now, but it is still open for the orientable closed surfaces of genus from two to four.

Let us consider nonorientable surfaces, in particular, the projective plane. Since the projective plane itself is not embeddable in $\mathbb{R}^{3}$, no map on the projective plane has a geometric realization. However, the surface obtained from the projective plane by removing a disk (i.e., a Möbius band) is embeddable in $\mathbb{R}^{3}$, and hence a triangulation on the Möbius band might have a geometric realization. For simple notation, we call a triangulation on the projective plane and that on the Möbius band a projective triangulation and a Möbius triangulation, respectively, throughout the paper.

In this paper, we discuss geometric realizations of Möbius triangulations. However, Brehm [4] found a Möbius triangulation with no geometric realization, which is shown in Fig. 1. (In Fig. 1, identify vertices with the same label.) Brehm essentially proved in [4] that for each of its spatial embeddings, the two disjoint 3-cycles 123 and 456 have a linking number at least two. (See [9] for the definition of the linking number.) However, two 3-cycles with straight segments in $\mathbb{R}^{3}$ have a linking number at most one. Hence we have the following: 
Fact 1 If a Möbius triangulation $G$ has a boundary 3-cycle $C$ and a 3-cycle $C^{\prime}$ disjoint from $C$ which forms an annular region with $C$, then $G$ has no geometric realization.

Bonnington and Nakamoto have proved the following theorem. Let $G$ be a projective triangulation, and let $f$ be a face of $G$. Let $G-f$ denote the Möbius triangulation obtained from $G$ by removing the interior of $f$.

Theorem 2 (Bonnington and Nakamoto [2]) Every projective triangulation $G$ has a face $f$ such that $G-f$ has a geometric realization.

In this paper, we would like to consider which faces $f$ of a given projective triangulation $G$ can be chosen so that $G-f$ is geometrically realizable. Suppose that $G$ has a face $f$ such that the boundary 3-cycle of $f$ has a nesting 3-cycle. In this case, we simply say that " $f$ has a nesting 3 -cycle." Then, by Fact $1, G-f$ has no geometric realization.

Our main theorem is as follows.

Theorem 3 Let $G$ be a projective triangulation, and let $f$ be a face of $G$. Then $G-f$ has a geometric realization if and only if $f$ has no nesting 3-cycle in $G$.

This theorem immediately implies Theorem 2, since we can always choose a face $f$ in a projective triangulation $G$ with no nesting 3-cycle. (Suppose that a face $g$ of $G$ has nesting 3-cycles. Choosing $C$ among them to outermost, i.e., $C$ has no nesting 3 -cycle, we can take a face $f$ in the 2 -cell region bounded by $C$ and sharing an edge with $C$; clearly, $f$ has no nesting 3-cycle.)

Moreover, Theorem 3 implies the following since, for any 3-cycle $C$ of a 4connected projective triangulation, Int $C$ contains no 3 -cycle other than $C$.

Corollary 4 Let $G$ be a 4-connected projective triangulation. Then, for any face $f$ of $G, G-f$ has a geometric realization.

A weaker version of Corollary 4 has already been proved by Chávez, Fijavž, Márquez, Nakamoto, and Suárez [5].

Theorem 5 (Chávez, Fijavž, Márquez, Nakamoto, and Suárez [5]) If G is a 5connected projective triangulation, then for any face $f$ of $G, G-f$ has a geometric realization.

Corollary 4 can be strengthened by introducing a notion of the cyclical $k$ connectivity. A graph $H$ is said to be cyclically $k$-connected if $H$ has no $k$-cut $S$ such that at least two components of $H-S$ have a cycle. We have the following, which is conjectured in [5].

Corollary 6 Let $G$ be a projective triangulation, and let $f$ be a face of $G$. Then, for any face $f$ of $G, G-f$ has a geometric realization if and only if $G$ is cyclically 4-connected. 


\section{Outline of the Proof}

In this section, we give an outline for the proof of Theorem 3. We prove our main result by using methods similar to those in $[2,5]$. The proof is split into two parts; the first part is graph-theoretical, and the second part is geometrical. It is the notion of exhibitions of maps that connects two different methods.

A plane map $R$ with a boundary walk $C$ of length $m \geq 3$ is said to be a near triangulation if $C$ is a cycle and if each inner face of Int $C$ is triangular. Let $R$ be a near triangulation, and let $C$ be the boundary cycle of $R$. Suppose that we are given an embedding of $C$ in $\mathbb{R}^{3}$ so that each edge is a straight-line segment, and that there exists a plane $P \subset \mathbb{R}^{3}$ such that the image of $C$ in $\mathbb{R}^{3}$ is projected to the plane $P$ as a convex polygon. Such an embedding of $C$ in $\mathbb{R}^{3}$ is called an exhibition.

Lemma $7[1,2]$ Let $R$ be a near triangulation, and let $C$ be the boundary of $R$. Suppose that an exhibition of $C$ in $\mathbb{R}^{3}$ is given. Then the exhibition of $C$ extends to a geometric realization of $R$ in the convex-hull of $C$ in $\mathbb{R}^{3}$.

Let $X$ be a map on a surface with each face bounded by a cycle. An embedding $\psi$ of $X$ into $\mathbb{R}^{3}$ is called an exhibition if

(i) for any face boundary $C$ of $X, \psi(C)$ is an exhibition,

(ii) for any two distinct faces $f$ and $f^{\prime}$ of $X$, the convex hulls of $\psi(f)$ and $\psi\left(f^{\prime}\right)$ intersect only at their common vertices and edges in the map.

An exhibition is a relaxed notion of a geometric realization since a geometric realization is an exhibition. In particular, if $G$ is a triangulation, then an exhibition of $G$ is equivalent to a geometric realization of $G$. The following is an important lemma for constructing a geometric realization of a map, and its proof can easily been obtained from the definition of exhibitions of near triangulations and maps.

Lemma 8 Let $G$ be a triangulation on a surface $F^{2}$, and let $X$ be a submap of $G$ which is a 2-cell embedding of $F^{2}$. If $X$ has an exhibition, then $G$ has a geometric realization.

In order to prove Theorem 3, we first prove the following, and Sect. 3 is devoted to it.

Lemma 9 Let $G$ be a projective triangulation, and let $f$ be a face of $G$ with no nesting 3-cycle. Then $G$ has a 4-cycle $C=v_{1} v_{2} v_{3} v_{4}$ separating $G$ into a Möbius triangulation $G_{M}$ and a near triangulation $G_{D}$ both of whose boundaries are $C$ such that

(i) $G_{M}$ has a submap $X$ with boundary cycle $C$ which is isomorphic to a subdivision of one of the nine maps shown in Fig. 2,

(ii) $G_{D}$ has a diagonal $v_{i} v_{i+2}$ for some $i$, and

(iii) $G_{D}$ contains $f$ such that $f=v_{i} v_{i+1} v_{i+2}$, or $v_{i} v_{i+2} \notin E(f)$ but $v_{i} \in V(f)$. 

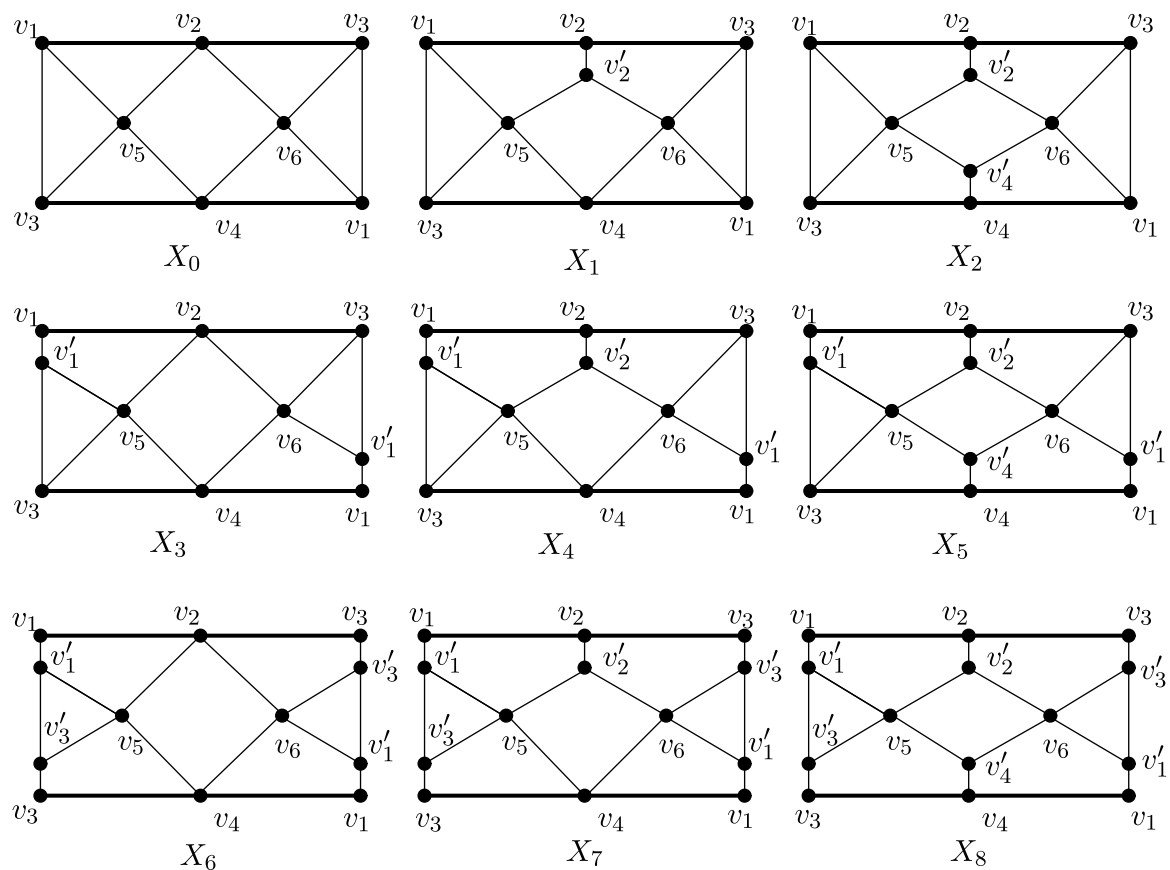

Fig. 2 Nine maps on the projective plane

In Sect. 4, we shall prove that $G_{M}$ in Lemma 9 has a geometric realization, and so does $G_{D}-f$ in the lemma to complete a geometric realization of $G-f$. In order prove that, we use computer programming.

In Sect. 5, we give a proof of Theorem 3 and concluding remarks.

\section{Contraction in Möbius 4-Triangulations}

Let $G$ be a triangulation on a surface $F^{2}$, and let $e$ be an edge of $G$. Contraction of $e$ is removing $e$, identifying the two endpoints of $e$, and replacing two pairs of parallel edges by two single edges. (For an edge $e$ contained in $\partial G$, the contraction of $e$ can be defined similarly except replacing one pair of parallel edges with a single edge.) Let $G / e$ denote the graph obtained from $G$ by contracting $e$. If $G / e$ is simple, then $e$ is said to be contractible. We always contract only a contractible edge. The inverse operation of the contraction of $e$ is a splitting of $v$, where $v$ is a vertex obtained from $e$ by its contraction.

Lemma 10 [8] Let $G$ be a triangulation on a surface $F^{2}$, and let $\Delta$ be a triangular region which is not a face. Then there is a contractible edge in the interior of $\Delta$.

Lemma 10 immediately implies the following: 
Lemma 11 Let $G$ be a triangulation on a nonspherical surface. Then $G$ has no contractible edge if and only if each edge of $G$ is contained in an essential 3-cycle.

Lemma 12 Let $G$ be a triangulation on a nonspherical surface, and let $C=$ $v_{1} v_{2} v_{3} v_{4}$ be a trivial 4-cycle of $G$. If $G$ has no contractible edge, then the interior of $C$ contains either a single diagonal or a single vertex. Furthermore, the latter happens if and only if the exterior of $C$ has two edges $v_{1} v_{3}$ and $v_{2} v_{4}$.

Proof If Int $C$ has a diagonal $v_{i} v_{i+2}, C$ satisfies Lemma 12 by Lemma 11 . So we suppose that $C$ has no diagonal in the interior of $C$. Then it is easy to see that the subgraph of $G$ induced by the inner vertices of $C$ is connected. So, if the interior of $C$ contains at least two vertices, then we can find an edge joining two inner vertices $w_{1}$ and $w_{2}$. By Lemma $11, w_{1} w_{2}$ is contained in an essential 3-cycle $D=w_{1} w_{2} x$ since $G$ has no contractible edge. However, since $w_{1}$ and $w_{2}$ are inner vertices of the interior of $C, D$ must be contained in $\operatorname{Int} C$. This contradicts that $D$ is essential. If the interior of $C$ contains a single vertex $v$, then $v$ is adjacent to $v_{1}, v_{2}, v_{3}, v_{4}$. Since $v v_{i}$ is not contractible for $i=1,2,3,4, v_{i}$ must be adjacent to $v_{i+2}$ in the exterior of $C$.

Let $R$ be a near triangulation with a boundary cycle $C$, and let $x, y \in V(C)$. A path jointing $x$ and $y$ and intersecting $C$ only at $x$ and $y$ is called an inner $(x, y)$-path or an inner path. We often use the following lemma proved in [2] to find a suitable subgraph in a near triangulation.

Lemma 13 [2] Let $R$ be a near triangulation with the boundary cycle $C$, and let $x, y \in V(C)$ with $x y \notin E(C)$. There is an inner $(x, y)$-path in $R$ if and only if there is no chord $p q$ for any $p, q \in V(C)-\{x, y\}$ such that $x, p, y$, and $q$ appear on $C$ in this cyclic order.

The Möbius band admits two types of essential simple closed curves: One is homotopic to the boundary of the Möbius band, and the other is homotopic to a center line of the Möbius band. The former separates the Möbius band, and the latter is nonseparating.

In this section, we deal with a Möbius triangulation $M$ whose boundary cycle has length exactly 4, which is called a Möbius 4-triangulation. (We sometimes let a Möbius 4-triangulation express a map on the projective plane with only one quadrilateral face, and all other faces are triangular.) Let $\partial M$ denote the boundary 4-cycle of $M$. Let $e$ be an inner edge of $M$, that is, an edge not lying on $\partial M$. We always consider the contractions of contractible inner edges. A Möbius 4-triangulation $G$ is said to be contractible to another Möbius 4-triangulation $G^{\prime}$ if $G$ is transformed in $G^{\prime}$ by a sequence of contractions of inner edges.

Let $X$ be the map on the Möbius band with six vertices and the boundary 4-cycle $v_{1} v_{2} v_{3} v_{4}$ such that each of two inner vertices is adjacent to all of $v_{1}, v_{2}, v_{3}$ and $v_{4}$ (see Fig. 3). A Möbius 4-triangulation $G$ with at least six vertices is said to be $X$ framed if $G$ has two inner vertices each of which is adjacent to all four vertices in the boundary. 
Fig. 3 The map $X$

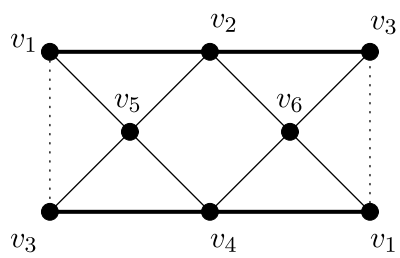

Lemma 14 Let $M$ be a Möbius 4-triangulation with the boundary cycle $C$. If $M$ has no 3-cycle homotopic to $C, M$ is contractible to a $X$-framed Möbius 4-triangulation with the boundary cycle $C$.

Proof Let $C=v_{1} v_{2} v_{3} v_{4}$. Suppose that $M$ has a trivial 3-cycle $D$ which is not a face boundary. Then int $D$ has a contractible edge $e$ by Lemma 10 . If $M / e$ has a 3 -cycle homotopic to $C, e$ is contained in a 4-cycle homotopic to $C$ in $M$, contrary to that $M$ has no 3-cycle homotopic to $C$ or $M$ is simple. Therefore $M / e$ has no 3cycle homotopic to $C$, and we may suppose that $M$ has no trivial separating 3-cycle. Suppose that $M$ has a 4-cycle $C^{\prime}=v_{1}^{\prime} v_{2}^{\prime} v_{3}^{\prime} v_{4}^{\prime}$ homotopic to $C$ but $C^{\prime} \neq C$. If $M$ has at least two such cycles, we take outermost one (i.e., no 4-cycle homotopic to $C$ in the region bounded by $C$ and $C^{\prime}$ ). By Menger's theorem [7], $M$ has a path $P_{i}$ from $v_{i}$ to $v_{i}^{\prime}$ for $i=1,2,3,4$ such that each $P_{i}$ is disjoint to $P_{j}$ for $j=1,2,3,4$, but $j \neq i$, since $M$ has no 3-cycle homotopic to $C$. There exists an edge $e=v_{i}^{\prime} p$ on $P_{i}$ adjacent to $v_{i}^{\prime}$ for some $i$ since $C \neq C^{\prime}$. If $e$ is a noncontractible edge, $e$ is contained in an essential 3-cycle $v_{i}^{\prime} p q$ since $M$ has no trivial separating 3-cycle. Moreover, since $M$ has no 3-cycle homotopic to $C, v_{i}^{\prime} p q$ is nonseparating. So $q$ must be contained in $C^{\prime}$, and $q \in V\left(P_{i-1}\right)$ or $V\left(P_{i+1}\right)$, contrary to the simpleness of $M$. Therefore we may suppose that $e$ is a contractible edge. If $M / e$ has a 3-cycle homotopic to $C, e$ is contained in a 4-cycle $v_{i}^{\prime} p q r$ homotopic to $C$ in $M$. Let $M^{\prime}$ be a triangulation on the Möbius band bounded by $C^{\prime}$. Since $C^{\prime}$ is outermost in $M, r$ must be an inner vertex of $M^{\prime}$, contrary to that $M$ has no 3-cycle homotopic to $C$ or $M$ is simple. Therefore if $M$ has a 4-cycle $C^{\prime}$ homotopic to $C$, we can contract edges of $M$ so that $C^{\prime}=C$ without 3-cycles homotopic to $C$. So we may suppose that $M$ has no 4-cycle homotopic to $C$ except for $C$, and we shall prove that if $M$ has no contractible edge, $M$ is an $X$-framed Möbius 4-triangulation.

Observe that $\operatorname{deg}_{M}\left(v_{i}\right) \geq 3$ for $i=1,2,3$, 4. (For otherwise, i.e., if $\operatorname{deg}_{M}\left(v_{2}\right)=2$ for example, then $M$ has a face $v_{1} v_{2} v_{3}$, and hence $v_{1} v_{3} v_{4}$ is a 3-cycle of $M$ homotopic to $C$, a contradiction.) If $\operatorname{deg}_{M}\left(v_{i}\right)=3$ for some $i$, say $N_{M}\left(v_{2}\right)=\left\{v_{1}, x, v_{3}\right\}$, then we have $x \notin\left\{v_{2}, v_{4}\right\}$. Hence we take $C^{\prime}=v_{1} x v_{3} v_{4}$ as a 4-cycle homotopic to $C$ in $M$, a contradiction. Therefore we may suppose that $\operatorname{deg}_{M}\left(v_{i}\right) \geq 4$ for $i=1,2,3,4$. So, if we let $v_{i} v_{i+1} l_{i}$ be the face of $M$ incident to the edge $v_{i} v_{i+1}$ for each $i$, then $l_{i}$ and $l_{i+1}$ are distinct. We shall prove that $l_{1}=l_{3}$ and $l_{2}=l_{4}$. So we may suppose that $l_{1} \neq l_{3}$ in $M$ by symmetry. Since $v_{1} l_{1}$ is not contractible, it is contained in an essential 3-cycle, say $C_{1}=v_{1} l_{1} x$, by Lemma 11 . Moreover, since $M$ has no 3-cycle homotopic to $C, C_{1}$ is nonseparating. Similarly, since $v_{3} l_{3}$ is not contractible by symmetry, $v_{3} l_{3}$ is contained in an essential nonseparating 3-cycle $C_{2}=v_{3} l_{3} y$. Since $C_{1}$ and $C_{2}$ are nonseparating cycles on the Möbius band and since $l_{1} \neq l_{3}, v_{1} \neq l_{3}$ $\left(l_{1} \neq v_{3}\right), v_{1} \neq v_{3}, x \neq v_{3}\left(v_{1} \neq y\right)$, and $x \neq l_{3}\left(l_{1} \neq y\right)$, we have $x=y$. See the left 

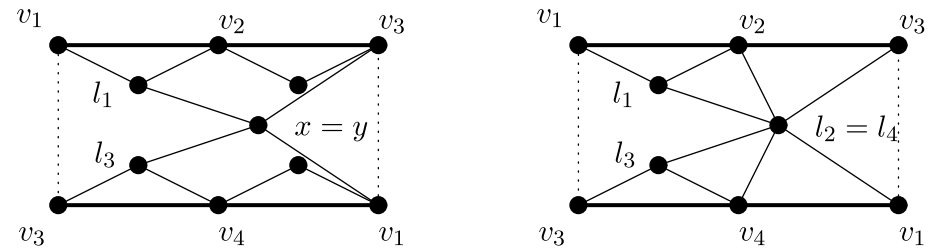

Fig. 4 The structure of $M$
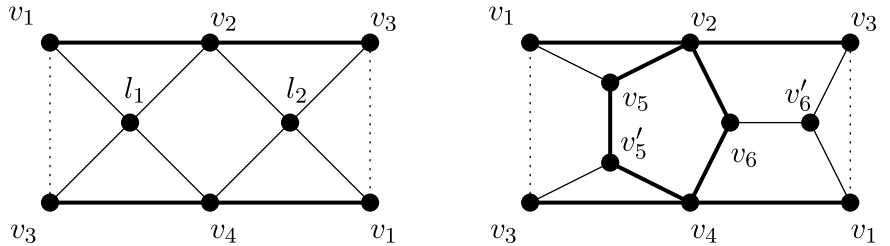

Fig. 5 The structure of $M$ (left) and an inner trivial cycle of $H$ (right)

in Fig. 4. By Lemma 12, each of the two quadrilateral regions $v_{1} x l_{3} v_{4}$ and $l_{1} v_{2} v_{3} y$ has a diagonal, which must be an edge $x v_{4}$ and $y v_{2}$, respectively. (For otherwise, we would have $\operatorname{deg}_{M}\left(v_{2}\right)=3$ or $\operatorname{deg}_{M}\left(v_{4}\right)=3$, contrary to that $\operatorname{deg}_{M}\left(v_{i}\right) \geq 4$ for $i=1,2,3,4$.) Moreover, we have $x=y=l_{2}=l_{4}$. See the right in Fig. 4 .

Now we focus on the edges $v_{2} l_{1}$ and $v_{4} l_{3}$. Similarly to the edge $v_{1} l_{1}$, if they are not contractible, they are contained in essential nonseparating 3-cycles $v_{2} l_{1} x^{\prime}$ and $v_{4} l_{3} y^{\prime}$, respectively. Since $v_{2}$ and $l_{1}$ have only one common neighbor other than $v_{1}$ and $l_{2}$, we have $x^{\prime}=v_{3}$. By the same argument, we have $y^{\prime}=v_{1}$. This is a contradiction since two 3-cycles $v_{2} l_{1} v_{3}$ and $v_{4} l_{3} v_{1}$ cannot exist simultaneously. So, we can contract inner edges until $l_{1}=l_{3}$ and $l_{2}=l_{4}$. See the left of Fig. 5. Hence $M$ is an $X$-framed Möbius 4-triangulation.

Let $M_{0}$ be an $X$-framed Möbius 4-triangulation with the boundary cycle $C=$ $v_{1} v_{2} v_{3} v_{4}$ and inner vertices $v_{5}, v_{6}$ adjacent to each vertex on $C$. Let $H_{0}$ be an $X$ frame of $M_{0}$. Consider a splitting of $v_{i}$ of $H_{0}$ into two vertices $v_{i}$ and $v_{i}^{\prime}$ of degree 3 . We always suppose that $v_{i}$, for $i=1,2,3,4$, lies on $C$. Let $H$, called a split $X$-frame, be a map with the boundary 4-cycle $C$ on the Möbius band obtained from $H_{0}$ by applying a sequence of either splitting of each $v_{i}$ or subdividing each edge. We call a vertex $v$ of $H$ whose degree is greater than 2 a node and a path in $H$ containing only two nodes as its endpoints a segment. Let $P(a, b)$ denote a path on a segment of $H$ with two endpoints $a, b$. If $a=b$, we suppose that $P(a, b)=\{a\}$. Moreover, $\left\{v_{i}, v_{i}^{\prime}\right\}$ is called a boundary split pair or boundary nodes if they arose by a splitting of $v_{i}$ on the boundary; otherwise it is called an inner split pair or inner nodes. Let $d$-segment be a segment of a split $X$-frame whose endpoints are a boundary node and an inner node. A trivial cycle of the split $X$-frame which contains four $d$-segments without segments on the boundary is called an inner trivial cycle. (For example, see the right of Fig. 5.) Observe that a split $X$-frame has two inner trivial cycles. 
Proof of Lemma 9 Let $D$ be a 3-cycle surrounding $f$. If $G$ has at least two such 3cycles, we choose the maximal one. Let $\tilde{f}$ denote a triangular region bounded by $D$. Observe that $G$ has no 3-cycle surrounding $\tilde{f}$ except for $\partial \tilde{f}$ since $G$ has no nesting 3 -cycle of $f$. Since $G$ is a triangulation, $G$ has three faces neighboring to $\tilde{f}$, among which we choose a face $f^{\prime}$ as follows. If $f$ has an edge $e$ such that $e \in E(\partial \tilde{f})$, we choose $f^{\prime}$ so that $e$ lies on $\partial\left(\tilde{f} \cup f^{\prime}\right)$. So we consider the case that $E(f) \cap$ $E(\partial \tilde{f})=\emptyset$. Let $u_{1}, u_{2}, u_{3}$ be vertices lying on $\partial \tilde{f}$. Since $f$ has no nesting 3-cycle, there exists a vertex $v$ of $f$ such that $v \in V(\partial \tilde{f})$. We may suppose that $v=u_{1}$. In this case, we choose $f^{\prime}$ whose boundary contains $u_{1} u_{3}$. Let $C$ be $\partial\left(\tilde{f} \cup f^{\prime}\right)$, and let $G_{M}$ (resp., $G_{D}$ ) be the Möbius triangulation (resp., the near triangulation) with boundary cycle $C$. Observe that $C$ satisfies the conditions (ii) and (iii) of Lemma 9.

We shall prove that $C$ satisfies the condition (i) of Lemma 9. Observe that, $G_{M}$ has no 3-cycle homotopic to $C$ by the definition of $C$. So $G_{M}$ is contractible to an $X$-framed 4-triangulation $G_{M 0}$ with the boundary cycle $v_{1} v_{2} v_{3} v_{4}$ by Lemma 14 . Therefore $G_{M}$ has a split $X$-frame.

Claim 1 There exists a split $X$-frame of $G_{M}$ with the boundary cycle $v_{1} v_{2} v_{3} v_{4}$ and no inner split pair.

Proof Let $H_{0}$ be an $X$-frame of $G_{M 0}$, and let $V\left(H_{0}\right)-V(C)=\left\{v_{5}, v_{6}\right\}$. Let $H$ be a split $X$-frame of $G_{M}$ with the boundary cycle $C=v_{1} v_{2} v_{3} v_{4}$ obtained from $H_{0}$ by a sequence of splitting of a vertex $v_{i}$ of $H_{0}$ into $v_{i}, v_{i}^{\prime}$ of degree 3 and subdividing edges. We may suppose that each segment of $H$ has no chord in $G_{M}$. (Otherwise we can take a shorter segment in $G_{M}$.) By the simpleness of $G_{M 0}$, we may suppose that the interior of $v_{5} v_{4} v_{6} v_{2}$ contains no chord from $v_{5}$ to $v_{6}$ in $G_{M 0}$. (Otherwise the interior of $v_{5} v_{3} v_{6} v_{1}$ does not contain it.) Therefore we may suppose that the interior of one inner trivial cycle of $H$ has no chord $x y$ such that $x \in V\left(P\left(v_{5}, v_{5}^{\prime}\right)\right)$ and $y \in V\left(P\left(v_{6}, v_{6}^{\prime}\right)\right)$ in $G_{M}$. Let $H^{\prime}$ be a split $X$-frame of $G_{M}$ with the boundary cycle $v_{1} v_{2} v_{3} v_{4}$. Let $u_{i}, u_{i}^{\prime}$ for $i=1,2,3,4$ be boundary nodes of $H^{\prime}$, and $u_{i}, u_{i}^{\prime}$ for $i=5,6$ be inner nodes of $H^{\prime}$. We always take $H^{\prime}$ in $G_{M}$ so that the interior of one inner trivial cycle has no chord from $x \in V\left(P\left(u_{5}, u_{5}^{\prime}\right)\right)$ to $y \in V\left(P\left(u_{6}^{\prime}, u_{6}\right)\right)$ and $P\left(u_{i}, u_{i}^{\prime}\right)$ for $i=5,6$ is as short as possible.

Suppose that each of $u_{i}$ for $i=1,2,3,4$ is a boundary split pair. (Otherwise we consider $u_{i}^{\prime}=u_{i}$ for $i=1,2,3,4$.) We shall prove that neither $u_{5}$ nor $u_{6}$ is a split pair of $H^{\prime}$. Suppose that $u_{5}$ is a split pair of $H^{\prime}$. Then, there are two cases of the splitting of $u_{5}$ as shown in Fig. 6 .
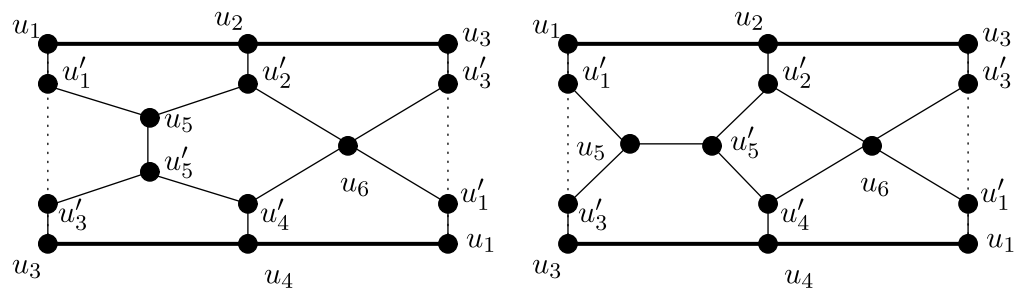

Fig. 6 A splitting of $u_{5}$ in $H^{\prime}$ 

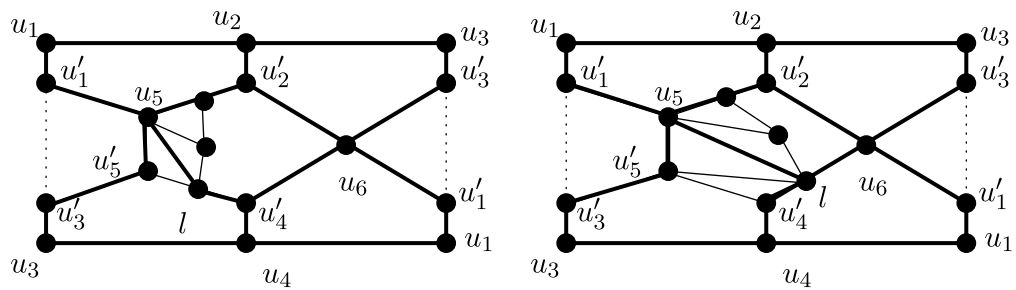

Fig. 7 Other split $X$-frames
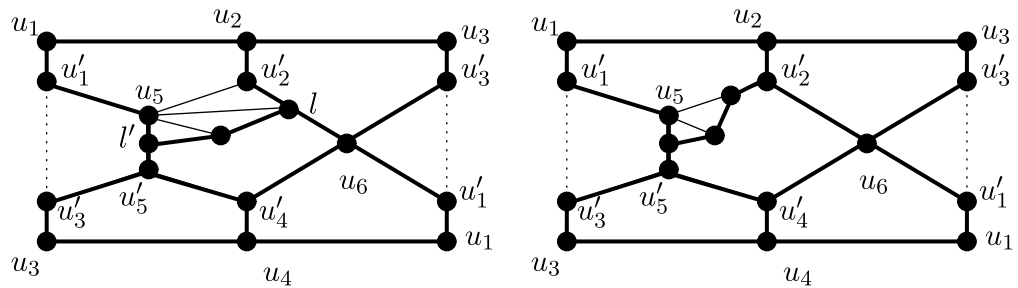

Fig. 8 Other split $X$-frames

First, we consider the case in the left-hand side of Fig. 6. There exist two inner trivial cycles $D_{1}, D_{2}$ in $H^{\prime}$. By the definition of $H^{\prime}$, we may suppose that $D_{1}=$ $P\left(u_{5}, u_{5}^{\prime}\right) P\left(u_{5}^{\prime}, u_{4}^{\prime}\right) P\left(u_{4}^{\prime}, u_{6}^{\prime}\right) P\left(u_{6}^{\prime}, u_{6}\right) P\left(u_{6}, u_{2}^{\prime}\right) P\left(u_{2}^{\prime}, u_{5}\right)$ and Int $D_{1}$ has no chord from $x \in V\left(P\left(u_{5}, u_{5}^{\prime}\right)\right)$ to $y \in V\left(P\left(u_{6}^{\prime}, u_{6}\right)\right)$ in $G_{M}$. Let us consider the neighbor of $u_{5}$ in Int $D_{1}$ on $G_{M}$. If Int $D_{1}$ has a chord from $u_{5}$ to $l$, where $l \in V\left(P\left(u_{4}^{\prime}, u_{5}^{\prime}\right)\right)-$ $\left\{u_{5}^{\prime}\right\}$ or $l \in V\left(P\left(u_{4}^{\prime}, u_{6}\right)\right)-\left\{u_{6}\right\}$, we can take other split $X$-frames, contrary to the assumption of $H^{\prime}$. (See Fig. 7.)

If Int $D_{1}$ has a chord from $u_{5}$ to $l$, where $l \in V\left(P\left(u_{2}^{\prime}, u_{6}\right)\right)-\left\{u_{6}\right\}$, then we can take an inner path from $l$ to $l^{\prime}$, where $l^{\prime}$ is adjacent to $u_{5}$ on $P\left(u_{5}, u_{5}^{\prime}\right)$, through the neighbor of $u_{5}$. If we regard $l^{\prime}$ as new $u_{5}$, we can take another split $X$-frame, contrary to the assumption of $H^{\prime}$. (See the left of Fig. 8.) If Int $D_{1}$ does not have such chords, we can take a path from $u_{5}^{\prime}$ to $u_{2}^{\prime}$ through the neighbor of $u_{5}$. So we can take another split $X$-frame, contrary to the assumption of $H^{\prime}$. (See the right of Fig. 8.)

In the right-hand side of Fig. 6, we consider the neighbor of $u_{5}$ in the interior of $P\left(u_{5}, u_{5}^{\prime}\right) P\left(u_{5}^{\prime}, u_{2}^{\prime}\right) P\left(u_{2}^{\prime}, u_{2}\right) u_{2} u_{1} P\left(u_{1}, u_{1}^{\prime}\right) P\left(u_{1}^{\prime}, u_{5}\right)$. By the same argument, we can obtain a contradiction. So $u_{5}$ of $H^{\prime}$ is not a split node. We can deal with $u_{6}$ similarly.

By Claim $1, G_{M}$ has a split $X$-frame with no inner split pair. Next, we shall prove that there exists a split $X$-frame with no inner split pair such that an inner trivial cycle has an inner path whose endpoints are two boundary nodes of it.

Claim 2 Let $D_{j}$ for $j=1,2$ be an inner trivial cycle of a split $X$-frame in $G_{M}$. There exists a split $X$-frame with the boundary $v_{1} v_{2} v_{3} v_{4}$ and no inner split pair such that one of Int $D_{j}$ has an inner path from a boundary node to another boundary node in $G_{M}$. 

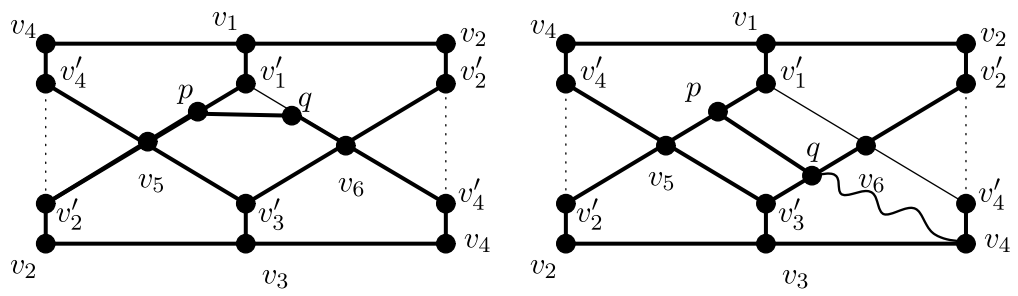

Fig. 9 Other split $X$-frames

Proof Let $H$ be a split $X$-frame of $G_{M}$ with the boundary $v_{1} v_{2} v_{3} v_{4}$ and no inner split pair. We may suppose that each segment of $H$ has no chord in $G_{M}$. (Otherwise we can take a shorter segment in $G_{M}$.) Let $v_{5}, v_{6}$ be inner nodes of $H$. We may suppose that $D_{1}=P\left(v_{1}^{\prime}, v_{5}\right) P\left(v_{5}, v_{3}^{\prime}\right) P\left(v_{3}^{\prime}, v_{6}\right) P\left(v_{6}, v_{1}^{\prime}\right)$. (If $v_{i}$ is not a split node, we regard $v_{i}$ as a node on $D_{j}$ for $j=1,2$.) We shall prove that one of Int $D_{j}$ contains an inner $\left(v_{j}^{\prime}, v_{j+2}^{\prime}\right)$-path in $G_{M}$. By the symmetry and simpleness of $G_{M}$, we may suppose that Int $D_{1}$ contains no chord $v_{5} v_{6}$. We take $H$ so that Int $D_{1}$ contains few chords in $G_{M}$ as possible. By Lemma 13 and symmetry, if Int $D_{1}$ contains no inner $\left(v_{1}^{\prime}, v_{3}^{\prime}\right)$-path, we may suppose that Int $D_{1}$ contains a chord $p q$ such that $p \in V\left(P\left(v_{5}, v_{1}^{\prime}\right)\right)-\left\{v_{1}^{\prime}, v_{5}\right\}$, $q \in V\left(P\left(v_{1}^{\prime}, v_{6}\right)\right)-\left\{v_{1}^{\prime}\right\}$ or $q \in V\left(P\left(v_{3}^{\prime}, v_{6}\right)\right)-\left\{v_{3}^{\prime}, v_{6}\right\}$.

If $q \in V\left(P\left(v_{1}^{\prime}, v_{6}\right)\right)-\left\{v_{1}^{\prime}\right\}$, we can regard $p$ as a new boundary node $v_{1}^{\prime}$, and we can take another split $X$-frame with no chord $v_{5} v_{6}$ in Int $D_{1}$ so that the number of chords contained in Int $D_{1}$ is fewer, a contradiction. (See the left of Fig. 9.) Therefore we may suppose that Int $D_{1}$ contains no chord $x y$ such that $x \in V\left(P\left(v_{5}, v_{1}^{\prime}\right)\right), y \in$ $V\left(P\left(v_{6}, v_{1}^{\prime}\right)\right)$ or $x \in V\left(P\left(v_{5}, v_{3}^{\prime}\right)\right), y \in V\left(P\left(v_{6}, v_{3}^{\prime}\right)\right)$.

If $q \in V\left(P\left(v_{3}^{\prime}, v_{6}\right)\right)-\left\{v_{3}^{\prime}, v_{6}\right\}$, let $P\left(v_{3}, v_{3}^{\prime}\right) P\left(v_{3}^{\prime}, v_{6}\right) P\left(v_{6}, v_{4}^{\prime}\right) P\left(v_{4}^{\prime}, v_{4}\right) v_{4} v_{3}=$ $D$. In this case, Int $D$ has no chord $r s$ such that $r \in V\left(P\left(v_{6}, q\right)\right)-\{q\}, s \in$ $V\left(P\left(q, v_{3}^{\prime}\right)\right)-\{q\}$ or $s \in V\left(P\left(v_{3}, v_{3}^{\prime}\right)\right)$ in $G_{M}$. (Otherwise the segment from $v_{6}$ to $v_{3}^{\prime}$ has a chord, or we can take another split $X$-frame with no chord $v_{5} v_{6}$ in Int $D_{1}$ so that the number of chords in Int $D_{1}$ is fewer, a contradiction.) Therefore Int $D$ has a path from $q$ to $v_{4}$ which does not intersect $V\left(P\left(v_{3}, v_{3}^{\prime}\right) \cup P\left(v_{3}^{\prime}, v_{6}\right)-\{q\}\right)$. If we regard $q$ as a $v_{6}$, we can take another split $X$-frame with no chord $v_{5} v_{6}$ in Int $D_{1}$ so that the number of chords in Int $D_{1}$ is fewer, a contradiction. (See the right of Fig. 9.) Hence Int $D_{1}$ has no chord $p q$, and we can take an inner $\left(v_{1}^{\prime}, v_{3}^{\prime}\right)$-path in $\operatorname{Int} D_{1}$.

By Claims 1 and 2, $G_{M}$ has a split $X$-frame $H$ with no inner split nodes and an inner $\left(v_{i}, v_{i+2}\right)$-path $P$ such that $P \cap H=P\left(v_{i}, v_{i}^{\prime}\right) \cup P\left(v_{i+2}, v_{i+2}^{\prime}\right)$. Then $G_{M}$ has one of sixteen subgraphs since for each boundary node, there exist two possibilities depending on whether it has a split pair or not. By the symmetry of $H \cup P, G_{M}$ has a subdivision of one of the nine maps shown in Fig. 2 as a subgraph. Therefore $C$ satisfies the condition (i) of the lemma. 


\section{Constructions of Geometric Realizations}

In this section, in order to prove Theorem 3, we shall construct geometric realizations and exhibitions of given maps. We first construct an exhibition of the nine maps $X_{0}, X_{1}, \ldots, X_{8}$ shown in Fig. 2 .

Lemma 15 For $i=0, \ldots, 8$, each $X_{i}$ has an exhibition.

Let $f_{1}, \ldots, f_{n}$ be the faces of $X_{i}$, and let $D_{j}$ be the boundary cycle of $f_{j}$ for each $j$. To prove Lemma 15 , by the definition of exhibitions, we have to arrange the vertices of $X_{i}$ in $\mathbb{R}^{3}$ so that

(i) the embedding of each $D_{j}$ is projected to some plane as a convex polygon, and

(ii) for any distinct $j, k \in\{1, \ldots, n\}$, two convex hulls $\left\langle D_{j}\right\rangle$ and $\left\langle D_{k}\right\rangle$ are disjoint except for their common points in $X_{i}$.

The following two computer programs, Programs 1 and 2, check that the coordinates of the vertices given really satisfy the above two conditions (i) and (ii), respectively.

Program 1 Let $A=a_{1} a_{2} \ldots a_{n}$ be a cycle embedded in $\mathbb{R}^{3}$ so that each edge $a_{i} a_{i+1}$ is a straight segment, where $a_{i} \in \mathbb{R}^{3}$ for each $i$.

Input: $\mathbb{R}^{3}$-coordinates of point sets $a_{1}, a_{2}, \ldots, a_{n}$ and a plane $F$.

Output: True if the projection of $A$ to $F$ is a convex polygon, and false otherwise.

The following is the procedures in Program 1.

Step 1. Let $A^{\prime}=a_{1}^{\prime} a_{2}^{\prime} \ldots a_{n}^{\prime}$ be a cycle on $F$ obtained by an orthogonal projection of $A=a_{1} a_{2} \ldots a_{n}$ to $F$, where $a_{i}^{\prime}$ corresponds to $a_{i}$ for each $i$. For $i=1,2, \ldots, n$, let $l_{i}$ be the straight line on $F$ through $a_{i}^{\prime}$ and $a_{i+1}^{\prime}$. If there exists $a_{i+2}^{\prime}$ lying on $l_{i}$ for some $i$, then return false. Otherwise go to Step 2.

Step 2. Let $F_{i}^{L}$ and $F_{i}^{R}$ be the two half-planes on $F$ separated by $l_{i}$, where we suppose that $F_{i}^{L}$ contains $a_{i+2}^{\prime}$. For each $i$, if there exists no $a_{j}^{\prime}$ with $j \neq i$ contained in $F_{i}^{R}$, then return true. Otherwise, return false.

Program 2 Let $A$ and $B$ be two point sets in $\mathbb{R}^{3}$. We check whether or not there exists a plane $F$ containing all points of $A \cap B$ and separating $A^{\prime}=A-(A \cap B) \neq \varnothing$ and $B^{\prime}=B-(A \cap B) \neq \emptyset$ in distinct sides. If there exists such $F$, then we say that $F$ is admissible.

Input: $\mathbb{R}^{3}$-coordinates of point sets $A=\left\{a_{1}, a_{2}, \ldots, a_{n}\right\}$ and $B=\left\{b_{1}, b_{2}, \ldots, b_{m}\right\}$, and a plane $F$ containing all points of $A \cap B$.

Output: True if $F$ is admissible, and false otherwise.

The following is the procedures in Program 2.

Step 1. Set $F^{+}$and $F^{-}$to be the two half-spaces in $\mathbb{R}^{3}$ separated by $F$, where some point $a_{i} \in A^{\prime}$ is contained in $F^{+}$. 
Step 2. If there is some $a_{i} \in F^{-}$, then return false. Otherwise go to Step 3 .

Step 3. If there is some $b_{i} \in F^{+}$, then return false. Otherwise return true.

The following is an easy observation.

Proposition 16 Let $X$ be a map on a surface, and let $f_{1}, \ldots, f_{n}$ be the faces of $X$. Suppose that the coordinates of all vertices of $X$ are given. If any two of $f_{i}$ and $f_{j}$ share at most one edge, then Programs 1 and 2 check whether the coordinates give an exhibition of $X$.

In Proposition 16, suppose that two faces $f_{1}$ and $f_{2}$ of $X$ share two vertices $x$ and $y$, but $x$ and $y$ are not adjacent in the boundary of $f_{1}$. Then, to start Program 2, we are given a plane $\pi$ containing $x$ and $y$ such that all vertices of $f_{1}$ and those of $f_{2}$ except $x$ and $y$ are located in two half-spaces separated by $\pi$. Though $f_{1}$ and $f_{2}$ share only $x$ and $y$, the convex polygon, say $P_{1}$, corresponding to $f_{1}$ and constructed by Program 1 shares a segment $x y$ on $\pi$ with the convex polygon, say $P_{2}$, corresponding to $f_{2}$. We can see that $P_{1}$ and $P_{2}$ share a segment $x y$ but $f_{1}$ and $f_{2}$ do not share an edge $x y$. Hence this is never an exhibition of $X$.

Proof of Lemma 15 For each map $X_{i}$, since any two faces share at most one edge, we can apply Proposition 16. We construct an exhibition of only $X_{2}$ since the remaining cases can be dealt similarly. (The cases for $X_{0}$ and $X_{1}$ are easier than that of $X_{2}$, since they have many triangular disks.)

Give the $\mathbb{R}^{3}$-coordinates of the vertices $v_{1}, v_{2}, v_{2}^{\prime}, v_{3}, v_{4}, v_{4}^{\prime}, v_{5}, v_{6}$ of $X_{2}$ as follows:

$$
\begin{array}{lll}
v_{1}=(12,-10,10), & v_{2}=(-10,10,-6), & v_{2}^{\prime}=(-9,10,-4), \\
v_{3}=(-16,-10,8), & v_{4}=(5,10,-12), & v_{4}^{\prime}=(4,10,-10), \\
v_{5}=(0,1,0), & v_{6}=(-1,20,0) . &
\end{array}
$$

See Fig. 10. We put $A, B, C, D, E, F, G$ to be the faces of $X_{2}$ as shown in the left. Then we can verify our coordinates of $X_{2}$ really gives an exhibition, as follows. We

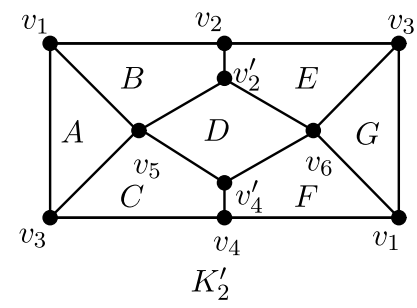

$v_{3}$

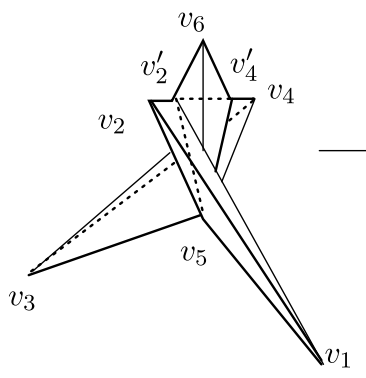

$B \cup C \cup D$

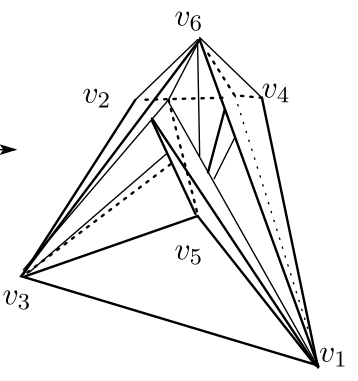

add $A, E, F$

Fig. 10 An exhibition of $X_{2}$ 
Table 1 Planes distinguishing two point sets

\begin{tabular}{|c|c|}
\hline The plane & Convex-hulls \\
\hline $11 x-128 y-154 z=-128$ & $A$ and $B$ \\
\hline $3 x+16 y+28 z=16$ & $A$ and $C$ \\
\hline $38 x+2 y-162 z=2$ & $A$ and $D$ \\
\hline $11 x-128 y-154 z=-128$ & $A$ and $E$ \\
\hline $190 x+10 y-217 z=10$ & $A$ and $F$ \\
\hline $11 x-128 y-154 z=-128$ & $A$ and $G$ \\
\hline $18 x+62 y+63 z=62$ & $B$ and $C$ \\
\hline $18 x+14 y-9 z=14$ & $B$ and $D$ \\
\hline $10 x-6 y-5 z=-130$ & $B$ and $E$ \\
\hline $95 x+65 y+19 z=585$ & $B$ and $F$ \\
\hline $90 x-101 y-420 z=-2110$ & $B$ and $G$ \\
\hline $54 x+106 y+117 z=106$ & $C$ and $D$ \\
\hline $28 x-48 y-28 z=-192$ & $C$ and $E$ \\
\hline $8 x+7 y+4 z=62$ & $C$ and $F$ \\
\hline $30 x-127 y-420 z=-257$ & $C$ and $G$ \\
\hline $11 x-6 y-5 z=-130$ & $D$ and $E$ \\
\hline $30 x+2 y+13 z=10$ & $D$ and $F$ \\
\hline $30 x-127 y-840 z=-2570$ & $D$ and $G$ \\
\hline $10 x-6 y-5 z=-130$ & $E$ and $F$ \\
\hline $90 x+179 y+840 z=3490$ & $E$ and $G$ \\
\hline $90 x-101 y-420 z=-2110$ & $F$ and $G$ \\
\hline
\end{tabular}

first see that by Program 1, each of the boundary cycles of $A, B, C, D, E, F, G$ can be projected to some plane as a convex polygon:

The projection $B$ to the plane $y=0$ is a convex polygon,

The projection of $C$ to the plane $z=0$ is a convex polygon,

The projection of $D$ to the plane $z=0$ is a convex polygon,

The projection of $E$ to the plane $-\frac{1}{1000} x-\frac{5017}{1000} y+\frac{8675}{1000} z=0$ is a convex polygon, The projection of $F$ to the plane $y=0$ is a convex polygon.

Secondly, by taking some suitable plane $F$ in Program 2, we can see that for any choice of two distinct faces, the convex hulls corresponding to them do not collide except their common points as in Table 1.

Therefore, our coordinates for $X_{2}$ can be verified to give an exhibition. The center of Fig. 10 shows three polygons corresponding to the convex-hulls of $B, C, D$ determined by our coordinates, and the right shows the body obtained from the convex hulls corresponding to $A, B, C, D, E, F$. Finally, to the body, we can easily add the disk corresponding to $G$ to get an exhibition of $X_{2}$.

For other $X_{i}$ 's, we can check that the following coordinates give an exhibition similarly to those for $X_{2}$ : We put movies of each exhibition of $X_{i}$ in the web site [12]. 
For $X_{0}$,

$v_{3}=(-16,-10,8)$,

$v_{6}=(-1,20,0)$.

For $X_{1}$,

$v_{2}^{\prime}=(-9,10,-4)$,

$v_{5}=(0,1,0)$,

For $X_{3}$,

$v_{2}=(-10,10,-6)$,

$v_{5}=(0,1,0)$,

For $X_{4}$,

$v_{2}=(-10,10,-6)$,

$v_{4}=(5,10,-12)$,

For $X_{5}$,

$v_{2}=(-10,10,-6)$,

$v_{4}=(5,10,-12)$,

$v_{6}=(-1,20,0)$.

For $X_{6}$,

$v_{2}=(-10,10,-6)$,

$v_{4}=(5,10,-12)$,

For $X_{7}$,

$v_{2}=(-10,10,-6)$,

$v_{3}^{\prime}=(-14,-10,10)$

$v_{6}=(-1,20,0)$.

For $X_{8}$,

$v_{2}=(-10,10,-6)$,

$v_{3}^{\prime}=(-14,-10,10)$,

$v_{5}=(0,1,0)$,

$$
v_{1}=(12,-10,10), \quad v_{2}=(-10,10,-6),
$$$$
v_{4}=(5,10,-12), \quad v_{5}=(0,1,0),
$$

$v_{1}=(12,-10,10), \quad v_{2}=(-10,10,-6)$,

$v_{3}=(-16,-10,8), \quad v_{4}=(5,10,-12)$,

$v_{6}=(-1,20,0)$.

$v_{1}=(12,-10,10), \quad v_{1}^{\prime}=(11,-10,20)$,

$v_{3}=(-16,-10,8), \quad v_{4}=(5,10,-12)$,

$v_{6}=(-1,20,0)$.

$v_{1}=(12,-10,10), \quad v_{1}^{\prime}=(11,-10,20)$,

$v_{2}^{\prime}=(-9,10,-4), \quad v_{3}=(-16,-10,8)$,

$v_{5}=(0,1,0), \quad v_{6}=(-1,20,0)$.

$v_{1}=(12,-10,10), \quad v_{1}^{\prime}=(11,-10,20)$,

$v_{2}^{\prime}=(-9,10,-4), \quad v_{3}=(-16,-10,8)$,

$v_{4}^{\prime}=(4,10,-10), \quad v_{5}=(0,1,0)$,

$v_{1}=(12,-10,10), \quad v_{1}^{\prime}=(11,-10,20)$,

$v_{3}=(-16,-10,8), \quad v_{3}^{\prime}=(-14,-10,10)$,

$v_{5}=(0,1,0)$,

$v_{6}=(-1,20,0)$.

$v_{1}=(12,-10,10), \quad v_{1}^{\prime}=(11,-10,20)$,

$v_{2}^{\prime}=(-9,10,-4), \quad v_{3}=(-16,-10,8)$,

$v_{4}=(5,10,-12), \quad v_{5}=(0,1,0)$,

$v_{1}=(12,-10,10), \quad v_{1}^{\prime}=(11,-10,20)$,

$v_{2}^{\prime}=(-9,10,-4), \quad v_{3}=(-16,-10,8)$,

$v_{4}=(5,10,-12), \quad v_{4}^{\prime}=(4,10,-10)$,

$v_{6}=(-1,20,0)$.

Lemma 17 Let $M$ be a Möbius triangulation with boundary cycle $v_{1} v_{2} v_{3} v_{4}$ containing one of the submaps $X_{0}, X_{1}, \ldots, X_{8}$. Unless $M$ has an edge $v_{1} v_{3}$ and $v_{2} v_{4}$, then $M$ has a geometric realization to which we can add a flat triangular disk $v_{j} v_{j+1} v_{j+2}$ with no intersection except their common points for each $j=1,2,3,4$. 
Fig. 11 Moving $v$ slightly to get $\hat{M}$

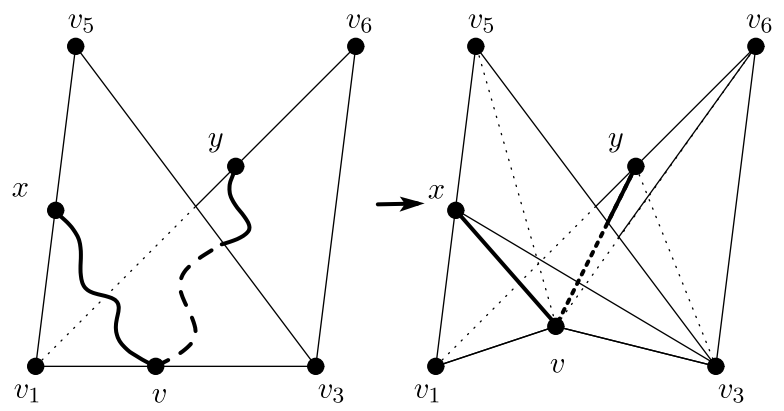

Proof Suppose that $M$ contains one of the submaps $X_{0}, \ldots, X_{8}$, say $X$. By Lemma 15 , each $X_{i}$ has an exhibition $\psi$, and hence $M$ has a geometric realization $\hat{M}$, where we take the coordinates of the vertices of $X$ contained in $\hat{M}$ by $\psi$ the same as in Lemma 15. Suppose that $X=X_{0}$ and we try to add a flat triangular disk $v_{1} v_{3} v_{4}$. Observe that the region, denoted by $D$, of $X$ formed by two triangular faces $v_{1} v_{5} v_{3}$ and $v_{1} v_{3} v_{6}$ and a triangular disk $\Delta=v_{1} v_{3} v_{4}$ or $\Delta^{\prime}=v_{2} v_{3} v_{4}$ added to it does not satisfy the assumption of Proposition 16. Let $\tilde{M}$ denote the map $M \cup \Delta$ or $M \cup \Delta^{\prime}$.

We first consider $\Delta$. We note that $D$ and $\Delta$ do not share an edge $v_{1} v_{3}$ in $\tilde{M}$. In this case, since $D$ has no chord $v_{1} v_{3}$ in $M$ by the assumption, we construct $D$ in a geometric realization of $\hat{M}$ as follows: Let $P$ be a shortest path in $D$ corresponding to an edge $v_{1} v_{3}$ of $X$. Then $P$ must be chordless in $M$. (For otherwise, we can choose a shorter one.) Since $D$ has no diagonal $v_{1} v_{3}, P$ has an inner vertex, say $v$, in $M$. Hence, by Lemma 13, $M$ has a path $P_{1}$ from $v$ to a vertex $x$ on the path corresponding to $v_{1} v_{5}$ or $v_{3} v_{5}$, and a path $P_{2}$ from $v$ to a vertex $y$ on the path corresponding to $v_{1} v_{6}$ or $v_{3} v_{6}$, where each of $x$ and $y$ is distinct from $v_{1}$ and $v_{3}$. Then $D$ can divided into four regions, say $D_{1}, D_{2}, D_{3}, D_{4}$. Move a position of $v$ in $\hat{M}$ slightly toward the interior of $\psi(D)$, where $\psi(D)$ is a tetrahedron in $\hat{M}$ corresponding to $D$. Note that a very small movement of $v$ in $\mathbb{R}^{3}$ does not yield an intersection of faces in $\hat{M}$. Hence we can take an exhibition of each of the regions $D_{1}, D_{2}, D_{3}$, and $D_{4}$ to get $\hat{M}$, and we can get a geometric realization of $\tilde{G}$. (For example, see Fig. 11.)

Now we secondly consider $\Delta^{\prime}=v_{1} v_{2} v_{4}$. Let $D^{\prime}$ be the region of $X$ bounded by $v_{2} v_{5} v_{4} v_{6}$. If $D^{\prime}$ has an internal path between $v_{2}$ and $v_{4}$, then we can do similarly as in the previous case. Otherwise, by Lemma $13, D^{\prime}$ has a chord $u v$ where $v_{2}, u, v_{4}$ and $v$ appear on the boundary of $D^{\prime}$ in this order. In this case, let $D_{1}^{\prime}$ and $D_{2}^{\prime}$ be the two regions obtained from $D^{\prime}$ by dividing along $u v$, and consider the exhibitions of $D_{1}^{\prime}$ and $D_{2}^{\prime}$. Since our coordinates of $D^{\prime}$ makes a tetrahedron in $\hat{M}$, these two exhibitions of $D_{1}^{\prime}$ and $D_{2}^{\prime}$ share only a segment $u v$, and the exhibition of $D_{1}^{\prime} \cup D_{2}^{\prime}$ does not intersect a straight segment in $\mathbb{R}^{3}$ between $v_{2}$ and $v_{4}$. (For example, see Fig. 12.)

Consequently, we can construct a geometric realization $\hat{M}$ avoiding a segment between $v_{1}$ and $v_{3}$ (or between $v_{2}$ and $v_{4}$ ) except their ends. Hence we can add each of $\Delta$ (or $\Delta^{\prime}$ ) to $X$ without intersections of faces, which can be checked by Programs 1 and 2 .

However, we cannot add each of the triangular disks $v_{1} v_{2} v_{3}$ and $v_{1} v_{2} v_{4}$ if we use the coordinates of each vertex of $X_{i}$ as in Lemma 15. In this case, we use the 

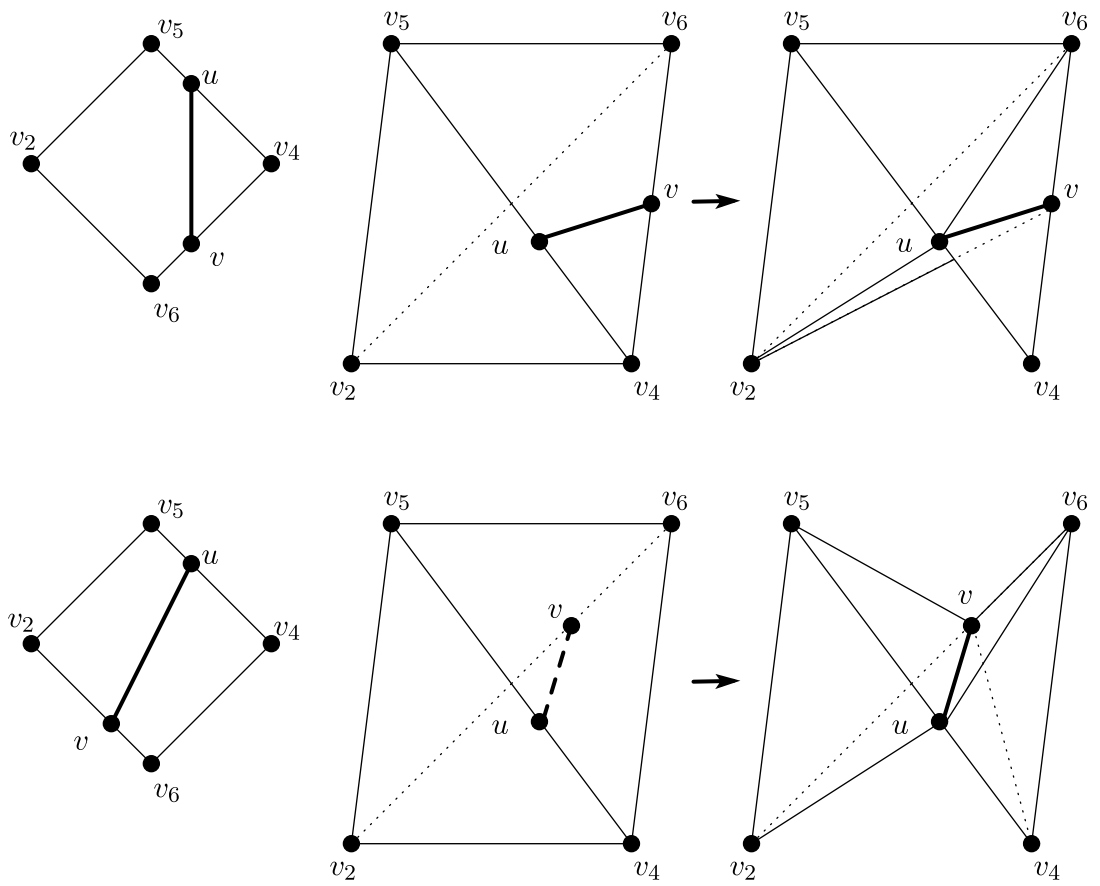

Fig. 12 An exhibition of $D^{\prime}$ and two exhibitions $D_{1}^{\prime} \cup D_{2}^{\prime}$

following coordinates of $X_{i}$, each of which is basically obtained from those in the proof of Lemma 15 by replacing the coordinate of each boundary node $v_{j}$ (resp. $v_{j}^{\prime}$ ) for $j=1,2,3,4$, with that of $v_{j+2}$ (resp. $v_{j+2}^{\prime}$ ) and the coordinate of each inner node $v_{j}$, for $j=5,6$, with that of $v_{j+1}$.

For $X_{0}$,

$$
v_{1}=(-16,-10,8), \quad v_{2}=(5,10,-12),
$$

$v_{3}=(12,-10,10)$,

$v_{4}=(-10,10,-6), \quad v_{5}=(-1,20,0)$,

$v_{6}=(0,1,0)$.

For $X_{1}$,

$$
v_{1}=(-16,-10,8), \quad v_{2}=(5,10,-12) \text {, }
$$

$v_{2}^{\prime}=(4,10,-10)$,

$v_{3}=(12,-10,10), \quad v_{4}=(-10,10,-6)$,

$v_{5}=(-1,20,0)$,

$v_{6}=(0,1,0)$.

For $X_{2}$,

$$
v_{1}=(-16,-10,8), \quad v_{2}=(5,10,-12) \text {, }
$$

$v_{2}^{\prime}=(4,10,-10)$,

$v_{3}=(12,-10,10), \quad v_{4}=(-10,10,-6)$,

$v_{4}^{\prime}=(-9,10,-4)$,

$$
v_{5}=(-1,20,0), \quad v_{6}=(0,1,0) \text {. }
$$

For $X_{3}$,

$$
v_{1}=(-16,-10,8), \quad v_{1}^{\prime}=(-14,-10,10) \text {, }
$$

$v_{2}=(5,10,-12)$,

$$
v_{3}=(12,-10,10), \quad v_{4}=(-10,10,-6) \text {, }
$$




$$
\begin{array}{lll}
v_{5}=(-1,20,0), & v_{6}=(0,1,0), & \\
\text { For } X_{4}, & v_{1}=(-16,-10,8), & v_{1}^{\prime}=(-14,-10,10), \\
v_{2}=(5,10,-12), & v_{2}^{\prime}=(4,10,-10), & v_{3}=(12,-10,10), \\
v_{4}=(-10,10,-6), & v_{5}=(-1,20,0), & v_{6}=(0,1,0), \\
\text { For } X_{5}, & v_{1}=(-16,-10,8), & v_{1}^{\prime}=(-14,-10,10), \\
v_{2}=(5,10,-12), & v_{2}^{\prime}=(4,10,-10), & v_{3}=(12,-10,10), \\
v_{4}=(-10,10,-6), & v_{4}^{\prime}=(-9,10,-4), & v_{5}=(-1,20,0), \\
v_{6}=(0,1,0) . & v_{1}=(-16,-10,8), & v_{1}^{\prime}=(-14,-10,10), \\
\text { For } X_{6}, & v_{3}=(12,-10,10), & v_{3}^{\prime}=(11,-10,20), \\
v_{2}=(5,10,-12), & v_{5}=(-1,20,0), & \\
v_{4}=(-10,10,-6), & v_{5}=(0,1,0) . \\
\text { For } X_{7}, & v_{1}=(-16,-10,8), & v_{1}^{\prime}=(-14,-10,10), \\
v_{2}=(5,10,-12), & v_{2}^{\prime}=(4,10,-10), & v_{3}=(12,-10,10), \\
v_{3}^{\prime}=(11,-10,20) & v_{4}=(-10,10,-6), & v_{5}=(-1,20,0), \\
v_{5}=(0,1,0) . & v_{2}^{\prime}=(4,10,-10), & v_{3}=(12,-10,10), \\
\text { For } X_{8}, & v_{4}=(-10,10,-6), & v_{4}^{\prime}=(-9,10,-4), \\
v_{2}=(5,10,-12), & \\
& & \\
v_{3}=(10,20), &
\end{array}
$$

Lemma 18 Let $M$ be a Möbius triangulation with boundary cycle $v_{1} v_{2} v_{3} v_{4}$ containing one of the submaps $X_{0}, \ldots, X_{8}$. Let $\hat{M}_{f}$ be a geometric realization of $M \cup f$ constructed by the coordinates in Lemma 15 or Lemma 17, where $f=v_{j} v_{j+1} v_{j+2}$ is a triangular disk for some $j \in\{1,2,3,4\}$. Then,

(i) for some point $p$ in $\mathbb{R}^{3}$, we can add two triangular disks $p v_{j} v_{j+2}$ and $p v_{i+2} v_{i+3}$ to the body of $\hat{M}_{f}$, and

(ii) for some points $q$ and $r$ in $\mathbb{R}^{3}$, we can add a tetrahedron qr $v_{i+2} v_{i+3}$, two triangular disks $q v_{i} v_{i+3}$ and $r v_{i} v_{i+2}$ to the body of $\hat{M}_{f}$.

Proof If $f=v_{1} v_{2} v_{3}$ or $v_{1} v_{3} v_{4}$, then we put $p=(0,0,-40), q=(-10,10,-6-$ $\left.\frac{1}{10}\right)$, and $r=(0,0,-40)$. If $f=v_{2} v_{3} v_{4}$ or $v_{1} v_{2} v_{4}$, then we put $p=(0,0,-40)$, $q=\left(11,-10+\frac{1}{10}, 8\right)$, and $r=(0,0,-40)$. Programs 1 and 2 verify Lemma 18 . 


\section{Proof of the Theorem and Concluding Remarks}

Proof of Theorem 3 Let $G$ be a projective triangulation, and let $f$ be a face of $G$. If $G$ has a nesting 3-cycle of $f$, then by Fact $1, G$ has no geometric realization, and so the necessity holds.

So we consider the sufficiency. Suppose that $f$ has no nesting 3-cycle in $G$. Then $G$ has a 4-cycle $C=v_{1} v_{2} v_{3} v_{4}$ satisfying (i), (ii), and (iii) of Lemma 9. Let $G_{M}$ (resp., $G_{D}$ ) be a Möbius triangulation (resp., a near triangulation) with the boundary cycle $C$. Since $C$ satisfies the condition (ii) of Lemma 9, $G_{D}$ has a diagonal $v_{i} v_{i+2}$, and the interior of $v_{i} v_{i+1} v_{i+2}$ contains $f$. For a simple notation, let $R$ and $R^{\prime}$ denote the 2-cell regions of $G$ bounded by $v_{i} v_{i+1} v_{i+2}$ and $v_{i} v_{i+2} v_{i+3}$, respectively. By Lemmas 15 and 17, $G_{M} \cup R^{\prime}$ has a geometric realization.

If $f=R$, then we are done, and hence we suppose $f \neq R$. If $E(f) \cup E(C) \neq \emptyset$, then $R$ has an inner vertex $p$ with $p \in V(f)$. In this case, there exists a path from $p$ to $v_{i+2}$ but disjoint from $v_{i}$ and $v_{i+1}$. If $E(f) \cup E(C)=\emptyset$, then $R$ has two inner vertices $q$ and $r$ with $q, r \in V(f)$. In this case, there exist two disjoint paths, say $P_{1}$ and $P_{2}$, from $q$ and $r$ to $v_{i+1}$ and $v_{i+2}$, not intersecting $v_{i}$. Without loss of generality, we may suppose that $P_{1}$ from $q$ to $v_{i+1}$ and $P_{2}$ is from $r$ to $v_{i+2}$. Therefore, by Lemma 18 , we can add all faces in $R$ except for $f$ to the body of a geometric realization of $G_{M} \cup R^{\prime}$ by taking suitable coordinates of $p$ or $q$ and $r$ in $\mathbb{R}^{3}$. Therefore, $G-f$ has a geometric realization.

Historically there are many papers concerning geometric realizations of orientable maps. For example, triangulations on the sphere and the torus has a geometric realization $[1,11]$. Moreover, there exists a triangulation on the orientable surfaces of genus $g \geq 5$ with no geometric realization $[3,10]$. However there are few papers concerning geometric realizations of nonorientable maps. We did not know the reason, but it might be by the Brehm's example, or because a nonorientable closed surface is not embeddable in $\mathbb{R}^{3}$.

Theorem 2 asserts that in a given projective triangulation $G$, if we choose a face $f$ carefully, then $G-f$ has a geometric realization. However, this theorem never describes a structure of geometrically realizable Möbius triangulations at all. In order to answer this question, Chávez, Fijavž, Márquez, Nakamoto, and Suárez [5] showed a result that a Möbius triangulation is geometrically realizable if it is a subgraph of a 5-connected projective triangulation. In proving the result, they used a technical argument for finding a $K_{6}$-minor with a good property in a projective triangulation $G$ with any face $f$ specified. However, this did not reach a complete characterization of a face $f$ with $G-f$ geometrically realizable. The current result (Theorem 3) completely solves the problem on geometric realization of a projective triangulation with one face removed, which has been considered in the two papers [2, 5].

Acknowledgement The authors would like to thank Kenta Ozeki for a lot of useful suggestions to improve the presentation and correctness of this paper.

\section{References}

1. Archdeacon, D., Bonnington, C.P., Ellis-Monaghan, J.A.: How to exhibit toroidal maps in space. Discrete Comput. Geom. 38, 573-594 (2007) 
2. Bonnington, P., Nakamoto, A.: Geometric realization of a projective triangulation with one face removed. Discrete Comput. Geom. 40, 141-157 (2008)

3. Bokowski, J., Guedes de Oliveira, A.: On the generation of oriented matroids. Discrete Comput. Geom. 24, 197-208 (2004)

4. Brehm, U.: A nonpolyhedral triangulated Möbius strip. Proc. Am. Math. Soc. 89, 519-522 (1983)

5. Chávez, M., Fijavž, G., Márquez, A., Nakamoto, A., Suárez, E.: Geometric realization of Möbius triangulations. SIAM J. Discrete Math. 23, 221-232 (2008)

6. Grünbaum, B.: Convex Polytopes. Interscience-Wiley, New York (1967)

7. Menger, K.: Zur allgemeinen Kurventheorie. Fundam. Math. 10, 95-115 (1927)

8. Negami, S.: Diagonal flips in triangulations of surfaces. Discrete Math. 135, 225-232 (1994)

9. Rolfsen, D.: Knot and Links. Math. Lecture Series, vol. 7. Publish or Perish, Berkeley (1976)

10. Schewe, L.: Satisfiability problems in discrete geometry. Technische Universität Darmstads (2007)

11. Steinitz, E.: Polyeder un Raumeinteilunger. Enzykl. Math. Wiss. 3(3A612), 1-139 (1922)

12. Tsuchiya, S.: http://tgt.ynu.ac.jp/tsuchiya.html 